\title{
SELF-ADAPTIVE FUZZY DECISION MAP MATCHING ALGORITHM BASED ON GIS BUFFER IN LCS
}

\author{
Yongjian Yang ${ }^{1}, \mathrm{Xu}$ Yang $^{2}$, Chijun Zhang ${ }^{1, *}$ \\ ${ }^{l}$ College of Computer Science and Technology, Jilin University, Changchun, Jilin, China, \\ 130012 \\ ${ }^{2}$ School of Electronics \& Information Engineering of Shenzhen Polytechnic, Shenzhen, \\ China, 518055 \\ * Corresponding author, Address: Chijun Zhang, College of Computer Science and \\ Technology, Jilin University, No. 2699 Qianjin Road Changchun, Jilin, China, 130012, \\ Tel: +86-0431-85168017,Email: yyj@jlu.edu.cn
}

\begin{abstract}
The wireless localization service is called Location-Based Services (LCS), is an value-added service provided by mobile communications network. This paper introduces a self-adaptive fuzzy decision algorithm based on GIS buffer. Candidate matching roads information is stored in GIS buffer. Through selfadaptive adjusting and fuzzy decision, the algorithm advances the differentiation degree between the right road and wrong. We can judge the matched road immediately, so it is a real-time algorithm and high accurate still. Changing the Coefficient-Value of measure factor by itself, the algorithm adjusts the weight of position information and direction information in judging the best matching road very well. In this way, the whole algorithm is optimized in many aspects.
\end{abstract}

Keywords: Location Based Service, Map Matching, Geography Information System, Fuzzy Decision

\section{INTRODUCTION}

The wireless localization service is called Location-Based Services (LCS), is an value-added service provided by mobile communications network, obtains the location information of mobile subscriber (for example latitude 
and longitude coordinates data) through some location technologies, provides for mobile subscriber or other people as well as the communications system, realizes each kind of the location relatives services. "Chinese Location-based Service Market Analysis" reported by Bo Tong Zhi Xin which is dedicated in telecommunication consultation indicated that the Chinese LCS service will enter the high speed growth phase in 2006 the second half year. The report thinks that the main reasons of the location service development so slow are that the LCS service technology, the content all quite complex, operation business and the SP preparation are insufficient, and faced with various development barrier, the cognition, the content, the technology, the terminal, the privacy, the backstage, cooperates and roams and so on.

This paper divides the LCS service correlation technology into three parts: Position technologies based on the mobile network, the core network side implementation technology about LCS services and the location service provider side implementation technology about LCS services. Through contrast current positioning accuracy and LCS service demand, no matter which one localization method uses, the mobile network all cannot guarantee stably provides real-time and precise location information. Along with the development of LCS services gradually, some service required high precise location information will be needed more precise requirement inevitably. Like the position accuracy which needed by navigation or track services is 30 meters and the system corresponding time in 5 seconds. There are two methods solve this problem, one method is that from access network side and the core network side adopts more precise position technology, but there is no feasible solution currently. Another solution is that joins a second position module in GIS engine in SP side, which function is implements map matching (Bernstein et al., 2002). The core of this paper is just the second positioning algorithm.

\section{MAP MATCHING ALGORITHM}

Map matching is a method of modifying location at the basis of software. The main idea is locating the position by connecting the position information with the road network in electronic map.

For different map matching algorithms, they are different only in the model and criterion, but the flow is same. 


\subsection{Set the area of matching error}

The first step of map matching is determining the error area (that is judgment area), so that we can get the information of candidates of matching road from the map database.

\subsection{Choose the suitable criterion for map matching}

After step (1), we have already got the candidates of matching road from map database. In this step, we begin matching with the defined matching criterion. It's the core of the whole matching algorithm, and different criterion will lead to different matching results.

\subsection{Judge the matching results synthetically}

GIS buffer technique is the basic function of GIS. We can get the geography information in buffer being in a distance to the positioned object. The core is GIS topological relation and advanced database links and query technique. The prior condition of buffer analysis is that the electronic map has complete topological relation and powerful database engine. Buffer analysis can extract the information of different layers within the buffer, which is useful for map matching. For example, we can analyze the road traffic and other geographic information in the circle of some point, some line and some area with buffer technique. For map matching, the task is searching the roads and nodes information near the location results in the system, and finding out the best matching point and matching it.

Above-mentioned algorithm is a typical map matching algorithm based on GIS buffer. We can see that it is suitable for the straight sparse highway. The solution for turning or intensive highway is to wait for presenting only one same value of highway, then position the current location using position data ahead. The flaws of this algorithm are as below:

The performance of real-time is not very well for solving turning or intensive highway section, even it would not respond for a long time.

The adaptability and the performance of dynamic adjusting are also not very well (George et al., 1999).

The reason for above is because of low positioning precision now, for example, GSM cell-phone positioning depends on the methods of TOA/TDOA or CELL-ID mainly, but the positioning error may reach tens of meters even more than one hundred meters. In this condition, it is very difficult to recognize the object turning real timely, and it is more difficult to match when it happens in intensive highway. 


\section{SELF-ADAPTIVE FUZZY DECISION MAP MATCHING ALGORITHM BASED ON GIS BUFFER}

The paper gives some supposing for introducing more explicitly.

First of all, the algorithm demands for more precise electronic map and position information. From the introduction at the head of the paper we can see that the precise of positioning has been improved in large extent with the integration of GPS and mobile network (Huang, et al., 2004). In this condition, the precise of positioning can reach within thirty meters. It has been possible to recognize the turning of highway. The special traffic electronic map is also needed to implement this algorithm. The precise of the electronic map must be limited less than $15 \mathrm{~m} 2$. Additionally, it is important to enrich the information of the electronic map, such as the integrality of the electronic map, the type of turning (right-angle, round square and crossroad), one-way street, left-forbidden and right-forbidden of the road and so on (Sinn et al., 2001).

Second, the positioning information refreshes every one second, and the data is saved in server. Dividing the positioned object into Fast-Moving Object (FMO) and Slow-Moving Object (SMO), Matching for FMO mainly adopts Straight Line Map Matching algorithm (SLMM) and Matching for SMO adopts Turning Map Matching algorithm (TMM). Generally speaking, FMO is more possible moving on the straight and wide highway, it would move longer distance in one second, so the position information showing on the electronic map can be refreshed in one second. SMO frequently runs in the area of turning, it could not move long distance only in one second, we can refresh the position information every three or four seconds, the position information in this interval is used as criterion for judging in what type of motion the object is moving. Then we can locate the object position in the electronic map by combining with the type of highway in the error area of the object.

On the basis of the supposing above, this paper introduces a judging model of candidate matching roads.

The DF (Direction Function) is defined as (Takagi et al., 1995):

$\mathrm{H}\left(h_{c}, h_{r}\right)=\cos \left(h_{c}-h_{r}\right)$

hc is introduced as the direction of the moving object.

$\mathrm{hr}$ is introduced as the direction of each candidate matching rode.

$\left(\left|h_{c}-h_{r}\right| \leq 90^{\circ}\right)$

The range of DF is: $(0,1]$

The PF (Position Function) is defined as (Takagi et al., 1995): 


$$
D\left(p_{c}, p_{r}\right)=\frac{1}{1+\left\|p_{c}-p_{r}\right\|^{2} / \sigma^{2}}
$$

$\mathrm{p}_{\mathrm{c}}=\left(x_{c}, y_{c}\right)$ is the position coordinate of the current moving object.

$\mathrm{p}_{\mathrm{r}}=\left(\mathrm{x}_{\mathrm{r}}, \mathrm{y}_{\mathrm{r}}\right)$ is the position coordinate of the point that is on the candidate matching road to which if current matching point matches.

$\sigma$ is defined as standard deviation of the position data. The value of is defined as 30 in this paper according to current positioning precision.

The range of $\mathrm{PF}$ is: $(0,1]$.

The function involved measurement factor of the positioning object is defined as:

$$
F=\alpha H\left(h_{c}, h_{r}\right)+\beta D\left(P_{c}, P_{r}\right)
$$

$\alpha, \beta$ is coefficient of measurement factor. The value of $\alpha, \beta$ is relative to the number of the cross roads which are fetched form GIS buffer. If there are several cross roads in GIS buffer, $\alpha$ will be bigger. Because the moving object is more possible to turn in this situation, the proportion of DF should be enlarged in function (3).

Measurement factor $\alpha, \beta$ is defined as below:

$$
\begin{aligned}
& \alpha=1+\cos ^{2}\left(\frac{h_{c}-h_{r}}{M+1}\right)-\cos ^{2}\left(h_{c}-h_{r}\right) \\
& \beta=1
\end{aligned}
$$

$M$ is the number of candidate matching roads in the GIS buffer. The weight of PF in function (3) of PF is invariable in the algorithm, so $\beta=1$. In order to improve the robustness of the algorithm, the connectivity amount the roads must be taken into account. So it is necessary to introduce the information of the previous matching points.

DF is modified as:

$$
H(K)=\cos \left(\frac{1}{N+1} \sum_{j=K-N}^{K}\left|h_{c_{j}}-h_{r_{j}}\right|\right)
$$

$\mathrm{PF}$ is modified as:

$$
D(K)=\frac{1}{1+\frac{1}{N+1} \sum_{\mathrm{i}=K-N}^{K} \frac{\left\|p_{c i}-p_{r i}\right\|^{2}}{\sigma^{2}}}
$$

The measurement factor is modified as:

$$
F(K)=\left(\frac{1}{N+1} \sum_{i=K-N}^{K} \beta_{i}\right) \cdot H(K)+\left(\frac{1}{N+1} \sum_{i=K-N}^{K} \alpha_{i}\right) \cdot D(K)
$$


$\mathrm{K}$ is the current candidate matching point. $\mathrm{N}$ is the number of previous matching points which are used to enrich the information of the current matching information. In order to decrease the complexity of the algorithm, $\mathrm{N}$ is always 2 or 3 . As a result of the joining of the previous $\mathrm{N}$ matching points, the judgment information of current candidate matching point is enriched. The robustness of the algorithm is improved and matching vibration is avoided at the same time.

For example, the matched roads of the matching points in center will switch between road A and road B. Certainly, we can also use the method of filter to solve this problem (Zhang et al., 2003). But the algorithm proposed in this paper solves the problem by the method of adding previous matching information to current judgment, the method is convenient and highefficient, it also makes the algorithm more universal. Additionally, considering some road is one-way, we define $\theta$ as the valid direction of the one-way street. Then the measurement factor of DF is modified as below:

$$
\alpha^{\prime}=\gamma \alpha
$$

$\gamma$ is a Step Function, it is defined as below:

$$
\begin{cases}\gamma=0 & \left|h_{c}-\theta\right| \geq 90^{\circ} \\ \gamma=1 & \left|h_{c}-\theta\right| \leq 90^{\circ}\end{cases}
$$

Finally, we define the Judging-Function as below:

$$
\mathrm{J}(\mathrm{K})=\max (\mathrm{F} 1(\mathrm{~K}), \mathrm{F} 2(\mathrm{~K}), \ldots \ldots . \mathrm{FC}(\mathrm{K}))
$$

Here, $\mathrm{C}$ is defined as the count of roads in the GIS buffer. The matched road judged by the algorithm is one of the candidate matching roads which makes $\mathrm{J}(\mathrm{K})$ reach maximum. The Self-Adaptive Fuzzy Decision Algorithm based on GIS buffer can be concluded as below:

1) Get the information of the candidate matching roads in GIS buffer (angle, coordinate, one-way street or not);

2) Get the matching information about the nearest $\mathrm{N}$ previous matching points;

3) Compute the measure values of every candidate matching roads through formulas of $\mathrm{H}(\mathrm{K})$ and $\mathrm{J}(\mathrm{K})$;

4) Confirm the matched road according $F(K)$ and $J(K)$.

\section{EMULATION TESTING AND DISCUSSION}

The following table is the object running logical traveling route in some region of road network: 
Table 1. The coordinate value of the object run

\begin{tabular}{cccc}
\hline Anchor point serial number & coordinate value & Anchor point serial number & coordinate value \\
\hline 1 & $(2.731,3.909)$ & 9 & $(2.631,3.772)$ \\
2 & $(2.710,3.907)$ & 10 & $(2.653,3.744)$ \\
3 & $(2.680,3.911)$ & 11 & $(2.668,3.717)$ \\
4 & $(2.650,3.906)$ & 12 & $(2.681,3.695)$ \\
5 & $(2.595,3.882)$ & 13 & $(2.709,3.684)$ \\
6 & $(2.588,3.849)$ & 14 & $(2.735,3.678)$ \\
7 & $(2.616,3.824)$ & 15 & $(2.758,3.680)$ \\
8 & $(2.620,3.800)$ & 16 & $(2.788,3.677)$ \\
\hline
\end{tabular}

According to the data of position, we can normalize the value of $F(K)$ in every second as membership of each candidate matching road and make in one figure.

Changing the Coefficient-Value of measure factor by itself, the algorithm adjusts the weight of position information and direction information in judging the best matching road very well. In this way, the whole algorithm is optimized in many aspects.

In the test, we find that there are several matching points whose positioning errors became larger when the moving object entered some road, but we can still get the matched road rightly. Obviously, the robustness of this algorithm has been improved after importing the matching information of the nearest $\mathrm{N}$ previous matching points.

We can also find that the real-time character of this algorithm is very well too. The membership-values of the right matching road and the wrong matching road change very fast when moving object enters one road from another. That is also attributed to importing the matching information of the forward matching points. Both of the right and wrong information are accumulated because of the previous information. So it advances the differentiation degree about the current matching information, then we can just spend little second in finding out the right road.

\section{ACKNOWLEDGEMENTS}

This work was supported by ZhuHai Technologies Plans Projects of China (Contract Number: PC200320007).

\section{REFERENCES}

Bernstein, D. and Kornhauser, A. Map matching for personal navigation assistants. 77th Annual meeting. The Transport Research Board, 2002, Jan 11-15, Washington D.C.

George Taylor and Geoffrey Blewitt. Virtual Differential GPS \& Road Reduction Filtering by

Map Matching. ION GPS'99, 14-17. 
Huang Yongfang, Yang Xinyong. A Map-Matching Algorithm Based on Adaptive Fuzzy Decision in Vehicle Navigation System [J]. Journal of Huaiyin Institute of Technology, 2004, 13(1).

Sinn Kim, Jong-Hwan Kim. Adaptive Fuzzy-network-based C-measure Map-matching Algorithm for Car Navigation System [J]. IEEE Transactions on Industrial Electronics, 2001, (4).

T. Takagi and M. Sugeno, Fuzzy Identification of System and its Application to Modeling and Control. IEEE Trans. Syst, Man and Cybern, 1995, 15(1).

Zhang M S. A new self-adaptive Kalman filtering method for GPS kinematic positioning. Journal of Central South University of Technology 2003, 34(5). 